\title{
Numerical simulation of seismic wave propagation in complex media by convolu- tional differentiator ${ }^{*}$
}

\author{
LI Xin-fü ${ }^{1), *}$ (李信富) LI Xiao-fan ${ }^{2)}$ (李小凡)
}

1) State Key Laboratory of Geological Processes and Mineral Resources, China University of Geosciences, Beijing 100083, China

2) State Key Laboratory of Lithospheric Evolution, Institute of Geology and Geophysics, Chinese Academy of Sciences, Beijing 100029, China

\begin{abstract}
We apply the forward modeling algorithm constituted by the convolutional Forsyte polynomial differentiator proposed by former worker to seismic wave simulation of complex heterogeneous media and compare the efficiency and accuracy between this method and other seismic simulation methods such as finite difference and pseudospectral method. Numerical experiments demonstrate that the algorithm constituted by convolutional Forsyte polynomial differentiator has high efficiency and accuracy and needs less computational resources, so it is a numerical modeling method with much potential.
\end{abstract}

Key words: seismic wave; numerical simulation; heterogeneous media; differential operator CLC number: P631 Document code: A

\section{Introduction}

The numerical algorithm and forward modeling for seismic wave propagation has long been a hot field in seismology. There have been many kinds of numerical algorithms for seismic forward modeling till now and the conventional methods commonly used are ray tracing method, integral equation method, finite difference method, finite element method, pseudospectral method and spectral-element method. However, aside from these, many important numerical methods such as cell automaton method (LI and HU, 1995), symplectic-geometry method (LUO et al, 2001) and convolutional differentiation operator method (Zhou and Greenhalgh, 1992; Zhou et al, 1993; ZHANG et al, 1996; DAI et al, 2005a, b) for seismic wave modeling are ignored by people, all these methods are good examples of the application in seismic wave forward modeling by means of some good ideas appeared in computational mathematics. The above-mentioned methods have their own property. Specially, in numerical computation, the design of the conventional convolutional differentiatial operator is based on the forward and inverse Fourier transform which entails a global operator, of length equal to the number of spatial samples (ZHANG et al, 1996). For the

\footnotetext{
* Received 2007-09-24; accepted in revised form 2008-05-06.

Foundation item: Open Fund of State Key Laboratory of Geological Processes and Mineral Resources, China University of Geosciences (GPMR0750) and National Natural Science Foundation of China (40437018).

^Author for correspondence: xinfuli@cugb.edu.cn
} 
reason of using a short operator, the conventional finite difference method has the merit of simple algorithm and fast computational speed, however, the numerical dispersion occurred in finite difference method is difficult to eliminate. The elimination of the numerical dispersion must be at the cost of much more computational time and the lower efficiency for the use of more grids. This analysis introduces the convolutional Forsyte polynomial differentiator (CFPD) into the seismic wave propagation forward modeling in complex heterogeneous geological media. Theoretical computation examples of 2-D seismic wavefield are given and the numerical results show that the algorithm can bring reliable outcomes with high precision and fast speed, and readily be a novel tool for the seismic modeling in more complex media.

\section{Basic theory of Forsyte polynomial differential operator}

According to the derivation of CHENG et al (2008), the expression of Forsyte polynomial differentiator can be expressed as

$$
\frac{\mathrm{d}}{\mathrm{d} x}=C_{1}+\sum_{k=2}^{N} C_{k} P_{k}^{\prime}(x)
$$

where

$$
C_{k}=\frac{\sum_{i=1}^{m} P_{k}\left(x_{i}\right)}{\sum_{i=1}^{m} P_{k}^{2}\left(x_{i}\right)}
$$

The discretized scheme of the equation (1) can be written as

$$
d_{1}(i \Delta x)=C_{1}+\sum_{k=2}^{N} C_{k} P_{k}^{\prime}(i \Delta x)
$$

Here, $i$ is the sampling index, $\Delta x$ is the sampling interval on $x$ axis. In order to eliminate the Gibbs effect and the Runge phenomena, the Gauss function has been applied to truncate the long operator, the short differential operator is shown below

$$
\begin{aligned}
& \hat{d}_{1}(i \Delta x)=\left\{\begin{array}{l}
(-1)^{i} d_{1}(i \Delta x) w(i) \\
-\hat{d}_{1}(j \Delta x)
\end{array} \quad i=-1,-2, \cdots,-m ; j=-1\right.
\end{aligned}
$$

The expression of the Gauss Function is

$$
w(i)=c e^{-a i \Delta x^{2}} \quad|i|=0,1,2, \cdots, m
$$

Where $m$ is the sampling length for each side, $c$ is a constant, $\alpha(0.1 \leq \alpha \leq 0.75)$ is attenuation factor.

The first-order differential operator with the length of nine-point used in this paper for the evaluation of the first-order velocity-stress wave equation has the optimal coefficients of -0.00234 , $0.00874,-0.0274,0.085,0.0,-0.085,0.0274,-0.00874,0.00234$. From the above-mentioned coefficients it can be seen that the differential operator is dissymmetry. Figure 1 shows the curve of amplitude versus sampling point for nine-point convolutional differentiatial operator, it can be seen that the amplitude can attenuate quickly.

CHENG et al (2008) tested the accuracy of the convolutional Forsyte polynomial differential operator with Gaussian function $f(x)=\mathrm{e}^{-\alpha^{2} x^{2}}, \alpha=1 / \Delta x, \Delta x=10$, the results demonstrate that the 


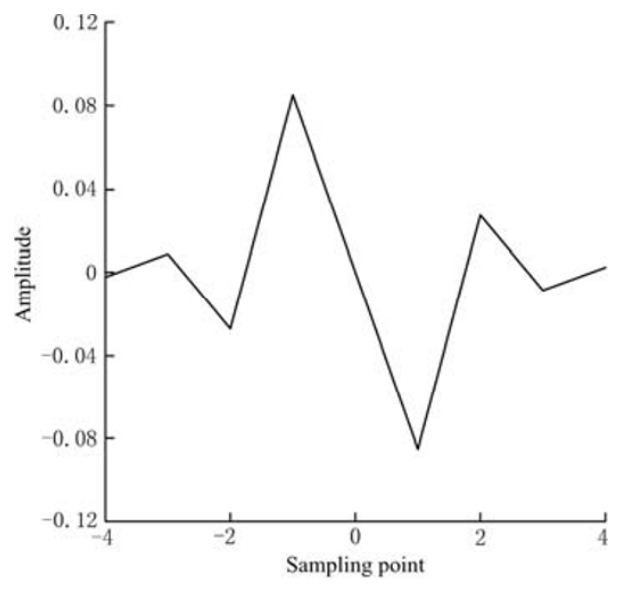

Figure 1 Amplitude versus sampling point for nine-point convolutional differentiator accuracy of the nine-point convolutional differential operator is much higher than that of the stagger-grid pseudospectral operator, and is as high as the 16th-order finite difference operator. So the nine-point convolutional differential operator method can be used for the simulation of seismic wave propagation with higher accuracy.

\section{Seismic wave propagation simu- lation}

\subsection{Basic equations and formulae}

The first-order velocity-stress elastic wave equation (assuming that the body force equals to zero) in 2-D inhomogeneous media can be expressed as follow

$$
\left\{\begin{array}{l}
\rho \frac{\partial v_{x}}{\partial t}=\frac{\partial \sigma_{x x}}{\partial x}+\frac{\partial \sigma_{x z}}{\partial z} \\
\rho \frac{\partial v_{z}}{\partial t}=\frac{\partial \sigma_{x z}}{\partial x}+\frac{\partial \sigma_{z z}}{\partial z} \\
\frac{\partial \sigma_{x x}}{\partial t}=(\lambda+2 \mu) \frac{\partial v_{x}}{\partial x}+\lambda \frac{\partial v_{z}}{\partial z} \\
\frac{\partial \sigma_{z z}}{\partial t}=(\lambda+2 \mu) \frac{\partial v_{z}}{\partial z}+\lambda \frac{\partial v_{x}}{\partial x} \\
\frac{\partial \sigma_{x z}}{\partial t}=\mu\left(\frac{\partial \sigma_{x}}{\partial z}+\frac{\partial \sigma_{z}}{\partial x}\right)
\end{array}\right.
$$

Here, $v_{x}$ and $v_{z}$ are the velocity components in $x$ and $z$ direction, respectively. And $\sigma_{x x}, \sigma_{x z}$ and $\sigma_{z z}$ are stress components. $\lambda$ and $\mu$ are Lame constants.

Assuming that $n, m, k$ are sampling points along $x, z$ and $t$ directions, $\Delta x, \Delta z, \Delta t$ are sampling intervals along $x, z$ and $t$ directions, and $m x, m z$ are the unilateral length of the operator along $x$ and $z$ directions, $\rho(n \Delta x, m \Delta z), \lambda(n \Delta x, m \Delta z), \mu(n \Delta x, m \Delta z)$ are the media density and Lame constants, $v_{x}$ and $v_{z}$ are $x$ and $z$ components of the seismic velocity. Based on the above-described parameters, equation (4) can be discretized as follows

$$
\begin{aligned}
v_{x}\left(n, m, k+\frac{1}{2}\right)= & v_{x}\left(n, m, k-\frac{1}{2}\right)+\frac{\Delta t}{\rho(n \Delta x, m \Delta z)}\left[\Delta x \sum_{i=-m x}^{m x} \hat{d}_{1}(i \Delta x) \sigma_{x x}(n-i, m, k)+\right. \\
& \left.\Delta z \cdot \sum_{j=-m z}^{m z} \hat{d}_{1}(j \Delta z) \sigma_{x z}(n, m-j, k)\right] \\
v_{z}\left(n, m, k+\frac{1}{2}\right)= & v_{z}\left(n, m, k-\frac{1}{2}\right)+\frac{\Delta t}{\rho(n \Delta x, m \Delta z)}\left[\Delta x \sum_{i=-m x}^{m x} \hat{d}_{1}(i \Delta x) \sigma_{x z}(n-i, m, k)+\right. \\
& \left.\Delta z \sum_{j=-m z}^{m z} \hat{d}_{1}(j \Delta z) \sigma_{z z}(n, m-j, k)\right]
\end{aligned}
$$




$$
\begin{gathered}
\sigma_{x x}(n, m, k+1)=\sigma_{x x}(n, m, k)+\Delta t \cdot\left\{[\lambda(n \Delta x, m \Delta z)+2 \mu(n \Delta x, m \Delta z)] \Delta x \sum_{i=-m x}^{m x} \hat{d}_{1}(i \Delta x) \cdot\right. \\
\left.v_{x}\left(n-i, m, k+\frac{1}{2}\right)+\lambda(n \Delta x, m \Delta z) \Delta z \sum_{j=-m z}^{m z} \hat{d}_{1}(j \Delta z) \cdot v_{z}\left(n, m-j, k+\frac{1}{2}\right)\right\} \\
\sigma_{z z}(n, m, k+1)=\sigma_{z z}(n, m, k)+\Delta t \cdot\left\{\lambda(n \Delta x, m \Delta z) \Delta x \sum_{i=-m x}^{m x} \hat{d}_{1}(i \Delta x) v_{x}\left(n-i, m, k+\frac{1}{2}\right)+\right. \\
\left.[\lambda(n \Delta x, m \Delta z)+2 \mu(n \Delta x, m \Delta z)] \Delta z \sum_{j=-m z}^{m z} \hat{d}_{1}(j \Delta z) \cdot v_{z}\left(n, m-j, k+\frac{1}{2}\right)\right\} \\
\sigma_{x z}(n, m, k+1)=\sigma_{x z}(n, m, k)+\Delta t \mu(n \Delta x, m \Delta z)\left[\Delta x \sum_{i=-m x}^{m x} \hat{d}_{1}(i \Delta x) v_{z}\left(n-i, m, k+\frac{1}{2}\right)+\right. \\
\left.\Delta z \sum_{j=-m z}^{m z} \hat{d}_{1}(j \Delta z) v_{x}\left(n, m-j, k+\frac{1}{2}\right)\right]
\end{gathered}
$$

The seismic source used here is the Ricker wavelet (YU, 1996), the boundary condition used here is the absorbing boundary condition put forward by Cerjan et al (1985) and Kosloff (1986).

\subsection{Numerical examples}

\subsubsection{Model I: Complex inhomogeneous model}

Figure 2 is a complex heterogeneous model, and Tabel 1 is the corresponding parameters. The model grid is $150 \times 120$, setting the grid point as the coordinate, the spatial interval is

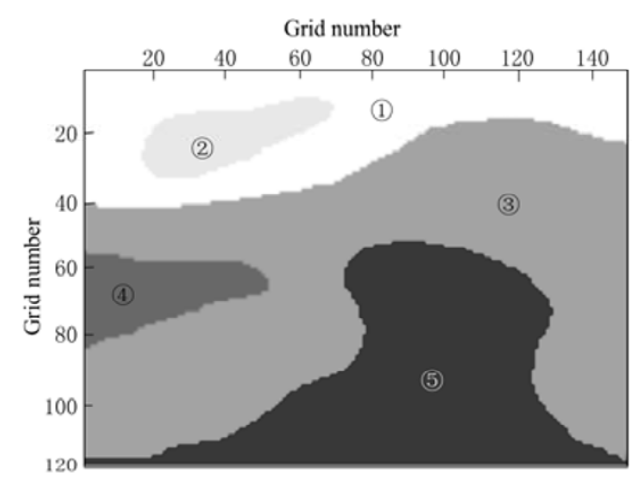

Figure 2 Complex heterogeneous model I $\Delta x=\Delta z=10 \mathrm{~m}$, time step is $\Delta t=1 \mathrm{~ms}$. The seismic source is located in media (1) with the coordinate $(75,15)$, the central frequency used here is $25 \mathrm{~Hz}$. Figure 3 show the snapshots obtained at the time of $400 \mathrm{~ms}$.
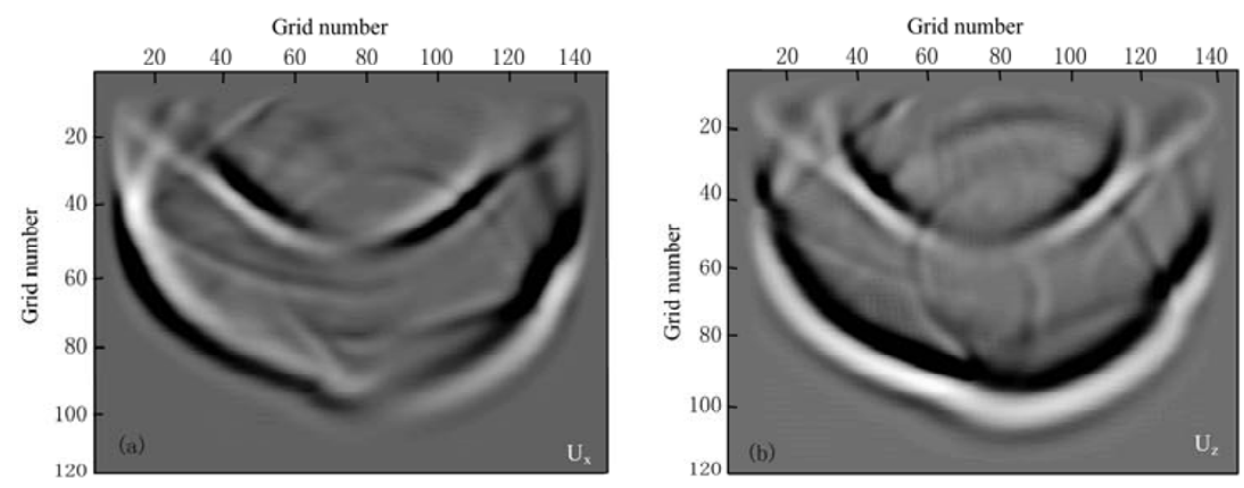

Figure 3 Snapshots of model I at the time of $400 \mathrm{~ms}$ (a) $x$-component; (b) $z$-component 
Table 1 Parameters for model I

\begin{tabular}{cccc}
\hline Media & $\rho / \mathrm{g} \cdot \mathrm{cm}^{-3}$ & $v_{\mathrm{P}} / \mathrm{m} \cdot \mathrm{s}^{-1}$ & $v_{\mathrm{S}} / \mathrm{m} \cdot \mathrm{s}^{-1}$ \\
\hline (1) & 1.9 & 3400 & 1950 \\
(2) & 2.0 & 3550 & 2100 \\
(3) & 2.2 & 3900 & 2200 \\
(4) & 2.3 & 4100 & 2300 \\
(5) & 2.5 & 4600 & 2650 \\
\hline
\end{tabular}

From Figure 3 it can be seen that the Forsyte polynomial differential operator method can be used to simulate the seismic wave propagation in specially complex inhomogeneous media.

\subsubsection{Model II: Marmousi model}

Marmousi model is a standard model with complex spatial velocity structure to test the seis-

mic image effect and to estimate the velocity accuracy, the spatial grid is $384 \times 122$ with grid interval $\Delta x=\Delta z=10 \mathrm{~m}$, we use the method proposed in this paper to simulate the seismic wave propagation in this model with the time interval of $\Delta t=1 \mathrm{~ms}$, the source location is at the point $(192,20)$, the central frequency of the wavelet is $25 \mathrm{~Hz}$. Aside from the P-wave velocity, other parameters are obtained on the basis of the P-wave velocity, such as that the S-wave velocity can be expressed as $v_{\mathrm{S}}=v_{\mathrm{P}} / \sqrt{3}$, and the density can be expressed as the Gardner formula $\rho=310 \times v_{\mathrm{P}}^{0.25}$. Figure 4 shows the Marmousi velocity model and Figure 5 show the snapshots at the time of $400 \mathrm{~ms}$.

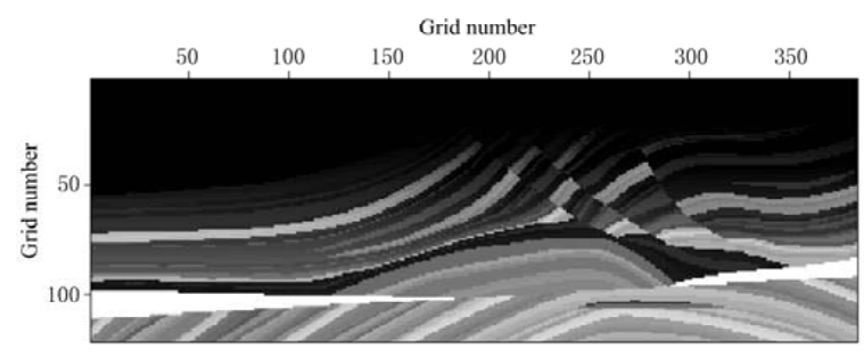

Figure 4 Marmousi velocity model
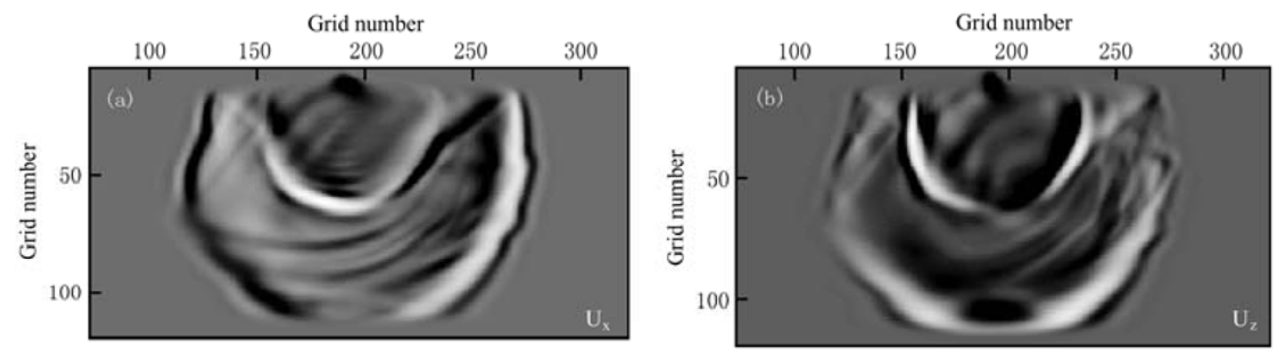

Figure 5 Snapshots for Marmousi model at the time of $400 \mathrm{~ms}$ (a) $x$-component; (b) $z$-component

From the snapshots obtained above we can see that there have obvious reflection and refraction on the interface and at the taper point.

\subsection{Time comparison with pseudospectral method (Zhou, 1993)}

Assuming that the 2-D spatial grid is $N \times N$, and the length of the differential operator is $M$. If we use pseudospectral method to simulate the seismic wave propagation, the complex multiplication times needed is $N^{2} \log _{2}^{N}$, while the method used this paper only need $M \times N^{2}$ times real multiplication. Obviously, the time ratio of latter to the former is $M / 4 \log _{2}^{N}$, so long as $M<4 \log _{2}^{N}$, the latter will have a higher efficiency than the former. 


\section{Discussion and conclusions}

This paper gives out a new application of the Forsyte polynomial differential operator to the seismic wave propagation simulation. The operator used in this paper is dissymmetry and can attenuate quickly. The introduction of the Gaussian window suppresses the Gibbs effect resulted from the truncation. Numerical results show that this method has the property of high speed and high efficiency. This method can be regarded as another new tool for the simulation of seismic wave propagation after the convolutional differential operator proposed by DAI et al $(2005 \mathrm{a}, \mathrm{b})$.

\section{References}

Cerjan C, Kosloff D, Kosloff R, et al. 1985. A nonreflecting boundary condition for discrete acoustic and elastic wave equation [J]. Geophysics, 50: 705-708.

CHENG Bing-jie, LI Xiao-fan, LONG Gui-hua. 2008. Seismic waves modeling by convolutional Forsyte polynomial differentiator method [J]. Chinese J Geophys, 51(2): 531-537 (in Chinese).

DAI Zhi-yang, SUN Jian-guo, ZHA Xian-jie. 2005a. Seismic wave field modeling with convolutional differentiator algorithm [J]. Journal of Jilin University (Earth Science Edition), 35(4): 520-524 (in Chinese).

DAI Zhi-yang, SUN Jian-guo, ZHA Xian-jie. 2005b. Seismic wave modeling with hybrid order convolution differentiator [J]. Computing Techniques for Geophysical and Geochemical Exploration, 27(2): 111-114 (in Chinese).

Kosloff R. 1986. Absorbing boundaries for wave propagation problems [J]. J Comput Phys, 63: 363-376.

LI You-ming and HU Jian-xing. 1995, Application of cellular automata approach to the study of seismic wave propagation [J]. Chinese $J$ Geophys, 38(5): 651-661 (in Chinese).

LUO Ming-qiu, LIU Hong, LI You-ming. 2001. Hamiltonian description and symplectic method of seismic wave propagation [J]. Chinese J Geophys, 44(1): 120-128 (in Chinese).

YU Shou-peng. 1996. Wide-band Ricker wavelet [J]. Oil Geophysical Prospecting, 31(5): 605-615 (in Chinese).

ZHANG Zhong-jie, TENG Ji-wen, YANG Ding-hui. 1996. Acoustic and elastic wave modeling by a convolutional differentiator [J]. Acta Seismologica Sinica, 9(1): 81-90.

Zhou B and Greenhalgh S.A. 1992. Seismic scalar wave equation modeling by a convolutional differentiator [J]. Bull Seism Soc Amer, 82(1): 289-303.

Zhou B, Greehalgh S.A., Zhe J. 1993. Numerical seismogram computations for inhomogeneous media using a short, variable length convolutional differentiator [J]. Geophysical Prospecting, 41: 751-766. 